\title{
DESENVOLVIMENTO DO LIMOEIRO 'VOLKAMERIANO' (Citrus volkameriana Pasq.) SUBMETIDO A DOSES DE PACLOBUTRAZOL E ÁCIDO GIBERÉLICO ${ }^{1}$
}

\author{
DALMO LOPES DE SIQUEIRA ${ }^{2}$, PAULO ROBERTO CECON ${ }^{3}$, LUIZ CARLOS CHAMHUM SALOMÃO $^{4}$
}

RESUMO-O trabalho foi desenvolvido com o objetivo de avaliar os efeitos de concentrações de paclobutrazol (PBZ) e ácido giberélico $\left(\mathrm{GA}_{3}\right)$ sobre o desenvolvimento de plantas de limoeiro 'Volkameriano' cultivadas em sacolas plásticas, contendo 2,5 $\mathrm{dm}^{3}$ de substrato. $O$ experimento foi montado em esquema fatorial $4 \times 2$, sendo quatro concentrações de $\operatorname{PBZ~}\left(0 ; 75 ; 150\right.$ e $225 \mathrm{mg}$ do i.a. planta $\left.{ }^{-1}\right)$ e duas de $\mathrm{GA}_{3}\left(0\right.$ e $20 \mathrm{mg}$ do i.a. $\left.\mathrm{L}^{-1}\right)$, no delineamento em blocos casualizados, com quatro repetições e cinco plantas por unidade experimental. Concentrações crescentes de PBZ reduziram o comprimento e diâmetro de caule, comprimento dos entrenós e área foliar, e aumentaram a massa foliar específica e as unidades SPAD. O GA reverteu a ação do PBZ. A aplicação de concentrações crescentes de PBZ, na presença de $\mathrm{GA}_{3}$, aumentou o diâmetro de caule até o valor máximo de $0,973 \mathrm{~cm}$, que foi alcançado com a concentração estimada de 90,0 mg planta ${ }^{-1}$ de PBZ. O aumento das concentrações de PBZ não alterou o número de folhas, enquanto o $\mathrm{GA}_{3}$, na ausência do PBZ, aumentou em $10 \%$ o número de folhas e reduziu em $17,93 \%$ o comprimento de caule.

Termos para indexação: Citrus volkameriana, porta-enxerto, retardadores de crescimento.

\section{GROWTH OF 'VOLKAMERIANO’ LEMMON TREE TREATED WITH PACLOBUTRAZOL AND GIBERELLIC ACID}

\begin{abstract}
This work was carried out to evaluate the effects of concentrations of paclobutrazol (PBZ) and giberellic acid (GA $)$ on the development of 'Volkameriano' lemon plants cultivated in recipients. The experiment was arranged in a $4 \times 2$ factorial scheme, with four concentrations of $\operatorname{PBZ}\left(0,75,150\right.$ and $225 \mathrm{mg}_{\text {of i.a. }}$ plant $\left.{ }^{-1}\right)$ and two concentrations of GA $\left(0\right.$ and $20 \mathrm{mg}_{3}$ i.a. $\left.\mathrm{L}^{-1}\right)$, in a randomized block design with four repetitions and 5 plants per experimental unit. Increasing PBZ concentrations reduced the length and stem

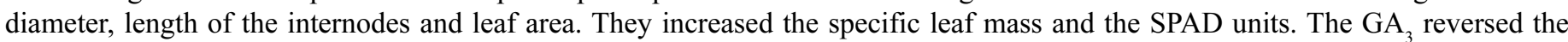
action of PBZ. The application of increasing concentrations of PBZ, in the presence of $\mathrm{GA}_{3}$, increased the stem diameter to the maximum value of $0,973 \mathrm{~cm}$ that was reached with the estimated PBZ concentration of $90.0 \mathrm{mg}$ plant-1. The leaves number was not influenced by PBZ concentrations, while $\mathrm{GA}_{3}$, in the absence of PBZ, increased the leaves number in $10 \%$ and reduced the stem length in $17.93 \%$.
\end{abstract}

Index terms: Citrus volkameriana, citrus rootstocks, plant growth retardants.

\section{INTRODUÇÃO}

O paclobutrazol (PBZ) atua inibindo a biossíntese de giberelinas (Rademacher, 1991), sendo usado como retardador de crescimento em grande número de culturas. O principal propósito do uso de PBZ é o controle de processos que são regulados por giberelinas endógenas.

Em citros, o PBZ tem mostrado sua efetividade como agente ananicante (Aron et al., 1985; Bausher \& Yelenosky, 1986), podendo ser utilizado para reduzir o crescimento excessivo de porta-enxertos durante a produção de mudas, principalmente de porta-enxertos vigorosos, como é o caso do limoeiro Volkameriano (Sorgoná et al., 2005). Neste caso, é preferível realizar aplicações foliares para não alterar o crescimento do sistema radicular (Bausher \& Yelenosky, 1986), devido à baixa mobilidade do produto pelo floema (Barret \& Bartuska, 1982; Tukey, 1983; Wang et al., 1986).

Duas aplicações foliares de PBZ a 100 e $500 \mathrm{mg} \mathrm{L}^{-1} \mathrm{em}$ limoeiro 'Volkameriano' reduziram o crescimento excessivo e indesejável do porta-enxerto em condições de viveiro. Entretanto nas concentrações de 200 e $500 \mathrm{mg}$. L-1, houve efeito residual do produto, prejudicando o desenvolvimento posterior das plantas. Em doses inferiores a $100 \mathrm{mg}$, a redução no crescimento foi menor, porém vantajosa, pois não houve efeito residual do produto (Hadlow \& Allan, 1989).

O PBZ, além de influenciar no florescimento das plantas, também modifica várias outras características cuja constatação

${ }^{1}$ (Trabalho 243-07). Recebido em: 05-10-2007. Aceito para publicação em:02-07-2008.

2Engenheiro Agrônomo, DS, professor do Departamento de Fitotecnia - Universidade Federal de Viçosa, 36570-000, Viçosa - MG - fone (31)38991349 - siqueira@ufv.br

${ }^{3}$ Engenheiro Agrônomo, DS, professor do Departamento de Informática - Universidade Federal de Viçosa, 36570-000, Viçosa - MG - tel (31)-38991781 - cecon@dpi.ufv.br

${ }^{4}$ Engenheiro Agrônomo, DS, professor do Departamento de Fitotecnia - Universidade Federal de Viçosa, 36570-000, Viçosa - MG - fone (31)-38991350 -1salomao@ufv.br 
e quantificação são difíceis em condições de campo, principalmente em plantas perenes e de porte elevado. Portanto, a aplicação em plantas mantidas em recipientes permite uma avaliação mais criteriosa dos efeitos do produto, inclusive nas raízes. Além disso, a escassez de trabalhos com uso de paclobutrazol em porta-enxertos de citros justifica a necessidade de estudos sobre o assunto.

Este trabalho foi desenvolvido com o objetivo de avaliar os efeitos de diferentes concentrações de PBZ na presença e ausência de ácido giberélico $\left(\mathrm{GA}_{3}\right)$, sobre desenvolvimento de seedlings de limoeiro 'Volkameriano'.

\section{MATERIAIS E MÉTODOS}

A pesquisa foi conduzida em casa de vegetação, no Setor de Fruticultura da Universidade Federal de Viçosa (UFV), situada em Viçosa - MG, no período de julho de 2002 a março de 2003.

Foram usadas plantas do limoeiro 'Volkameriano' (Citrus volkameriana) mantidas em sacolas plásticas, contendo $2,5 \mathrm{dm}^{3}$ do substrato composto por $30 \%$ de esterco bovino curtido, $30 \%$ de solo argiloso, $20 \%$ de areia e $20 \%$ de vermiculita, com fertilização à base de $6,0 \mathrm{~kg}$ de superfosfato simples; $2,00 \mathrm{~kg}$ de calcário dolomítico; $85 \mathrm{~g}$ de sulfato de cobre; $34 \mathrm{~g}$ de sulfato de zinco; $37 \mathrm{~g}$ de sulfato de manganês; $48 \mathrm{~g}$ de sulfato de ferro; 0,25 $\mathrm{g}$ de molibdato de amônio, e $0,75 \mathrm{~g}$ de ácido bórico por metro cúbico de substrato.

$\mathrm{O}$ experimento foi montado em esquema fatorial $4 \times 2$, com quatro concentrações de PBZ $(0 ; 75 ; 150$ e $225 \mathrm{mg}$ do ingrediente ativo planta $\left.{ }^{-1}\right)$ e duas de $\mathrm{GA}_{3}(0$ e $20 \mathrm{mg}$ do ingrediente ativo $\mathrm{L}^{-1}$ ). $\mathrm{O}$ delineamento usado foi o de blocos casualizados, com quatro repetições e cinco plantas por unidade experimental. Os tratamentos foram aplicados quando as plantas completaram seis meses de idade e altura média de $32 \mathrm{~cm}$.

O paclobutrazol (PACHLOBUTRAZOL $100 \mathrm{CE}-10 \%$ i.a.) foi aplicado na forma de solução, individualmente no substrato de cada planta, em aplicação única, com volume de $50 \mathrm{~mL}$.

O ácido giberélico (PRO-GIBB, 10\% i.a.) foi aplicado em quatro pulverizações foliares, com intervalos de dez dias, até o ponto de escorrimento. Foi adicionado espalhante adesivo à base de copolímero de poliéster e silicone, na razão de $1 \mathrm{~cm}^{3} \mathrm{~L}^{-1}$ à solução de $\mathrm{GA}_{3}$ e da água destilada, aplicada nos tratamentos sem $\mathrm{GA}_{3}$. A aplicação do $\mathrm{GA}_{3}$ teve início no mesmo dia da aplicação do PBZ.

No período de duração do experimento, foram tomadas temperaturas máximas e mínimas diárias, no interior da casa de vegetação. A média das máximas foi de $36,1 \pm 3,1^{\circ} \mathrm{C}$ e a média das mínimas foi de $19,0 \pm 1,2^{\circ} \mathrm{C}$.

Aos 50 dias após o início da aplicação dos tratamentos, foram avaliados o comprimento e o diâmetro do caule (dois $\mathrm{cm}$ acima do coleto da planta), o número de folhas, o comprimento médio dos entrenós, a intensidade da cor verde nas folhas, em unidades SPAD (SPAD-502 (Soil-Plant Analysis Development), Section Minolta Câmera Co. Ltd. Japão), a área foliar por planta (Area Meter Model LI - 3100) e a massa foliar específica (quociente da massa da matéria fresca das folhas, em gramas, e o valor de área foliar, $\mathrm{em}^{2}$ ).
Os dados foram analisados usando as análises de variância e regressão. Independentemente da significância da interação $\mathrm{GA}_{3}$ X PBZ, optou-se pelo seu desdobramento, para estudar os efeitos das concentrações de PBZ na presença ou ausência de $\mathrm{GA}_{3}$.

Os modelos foram escolhidos com base na significância dos coeficientes de regressão, utilizando-se do teste de $t$, a $5 \%$ de probabilidade, no coeficiente de determinação $\left(\mathrm{R}^{2}=\right.$ SQRegressão/SQPBZ) e no potencial para explicar o fenômeno biológico.

\section{RESULTADOS E DISCUSSÃO}

O resumo dos resultados das análises de variância encontra-se na Tabela 1. O comprimento do caule e dos entrenós do limoeiro 'Volkameriano' decresceu linearmente com o aumento das concentrações de PBZ (Figuras 1A e 2B), tanto na presença como na ausência de $\mathrm{GA}_{3}$.

Para o comprimento do caule, as reduções foram de $42,09 \%$ na ausência de $\mathrm{GA}_{3}$, contra $8,30 \%$ na presença. O GA sem PBZ, promoveu alongamento do caule em 17,93\%. O comprimento dos entrenós foi reduzido em $39,76 \%$ na ausência de $\mathrm{GA}_{3}$ e em $11,33 \%$ na presença, considerando o controle e a maior concentração de PBZ. Yelenosky et al. (1995) também observaram redução no comprimento e diâmetro do caule em plântulas de vários porta-enxertos de citros, que receberam aplicação via solo de PBZ nas concentrações de100 e $250 \mathrm{mg}$ planta $^{-1}$.

O número de folhas das plantas de limoeiro 'Volkameriano' foi, em média, $10 \%$ maior na presença de $\mathrm{GA}_{3}$, mas não foi afetado pelo PBZ (Figura 2A). A falta de efeito do PBZ sobre o número de folhas pode ser atribuída às concentrações aplicadas, que foram baixas quando comparadas às usadas por outros autores. Vu \& Yelenosky (1992) relataram que o tratamento com PBZ reduziu o tamanho e o número total de folhas, nas concentrações de 100; 250 e $500 \mathrm{mg} \mathrm{planta}^{-1}$ de PBZ.

Vários autores afirmaram que o efeito do PBZ sobre a produção de novas folhas depende da concentração aplicada. Em altas concentrações, a produção de folhas é reduzida, enquanto em concentrações baixas o número de folhas praticamente não é alterado (Young, 1983; Braun \& Garth, 1986; Jiao et al., 1986; Sankhla et al., 1986).

O comprimento dos entrenós foi reduzido com o aumento das concentrações de PBZ, tanto na presença como na ausência de $\mathrm{GA}_{3}$, com maior redução na ausência (Figura 2B). A redução do comprimento dos entrenós é a resposta morfológica mais conhecida causada pelo PBZ em caules de plantas (Quinlan, 1981). Nos citros, uma de suas utilizações é no controle do crescimento excessivo das plantas (Aron et al., 1985). Segundo Delgado et al. (1986), as concentrações de cinco e $10 \mathrm{~g}$ de i.a. por planta, via solo, diminuíram o comprimento das brotações em tangerinas 'Frost Dancy' (C. reticulata Blanco).

O efeito do paclobutrazol é variável, não só em função da concentração aplicada, mas também em função da espécie ou variedade e do tempo transcorrido desde a aplicação. Lidón et al. (2001) observaram que o PBZ reduziu o comprimento dos entrenós 
de forma mais acentuada na laranjeira 'Azeda' (C. aurantium) do que em C. macrophylla. Nesta última espécie, a redução só ocorreu com as concentrações máximas (5.000 $\mathrm{mg}$ planta $^{-1}$ do i.a.). Os sintomas foram observados depois de transcorrido um período de tempo maior do que o observado para a laranja 'Azeda'. A redução do crescimento dos ramos é atribuída à diminuição da quantidade total de açúcares disponíveis nos mesmos, induzida pela ação do PBZ (Mehouachi et al., 1996).

Redução no comprimento dos ramos, obtida pelo encurtamento dos entrenós, sem reduções significativas no número de folhas, é a forma mais eficiente de controlar o crescimento da planta sem prejudicar a eficiência fotossintética, uma vez que o duplo papel (síntese hormonal e nutricional) das folhas nos processos envolvidos com o desenvolvimento das plantas é bastante conhecido (Monselise \& Goldschmidt, 1982).

Embora não tenha afetado o número de folhas, a área foliar por planta foi reduzida pelas doses de PBZ na ausência de $\mathrm{GA}_{3}$ Na presença, o efeito do PBZ foi neutralizado (Figura 3A). Isso indica que o tamanho das folhas foi reduzido em função das doses de PBZ.

Resultados semelhantes foram encontrados por Swietlik \& Miller (1983), DeJong \& Doyle (1984), Wood (1984), Sankhla et al. (1985) e Steffens et al. (1985). Segundo Sankhla et al. (1985), Steffens et al. (1985) e Williamson et al. (1986), a redução na área foliar devido à aplicação de PBZ é, proporcionalmente, maior que a redução da massa da matéria seca foliar. Isso pode ocorrer, pois a redução no tamanho é compensada pelo aumento na espessura da folha.

A massa foliar específica (MFE) (Figura 3B) aumentou $7,45 \%$ com as crescentes concentrações de PBZ, na ausência de $\mathrm{GA}_{3}$, porém, na presença de $\mathrm{GA}_{3}$, o $\mathrm{PBZ}$ não apresentou nenhum efeito. $\mathrm{O} \mathrm{GA}_{3}$, na ausência de $\mathrm{PBZ}$, reduziu a MFE em 5,11\%.

Como o PBZ não afetou o número de folhas, diminuiu a área foliar e aumentou sua massa específica, provavelmente, houve aumento da espessura das folhas. Trabalhando com nozpecã (Carya illinoensis), Wood (1984) relatou que o paclobutrazol aumentou a espessura das folhas. Resultados semelhantes também foram encontrados por Sankhla et al. (1985), Steffens et al. (1985) e Williamson et al. (1986) com citros.

Por outro lado, Vu \& Yelenosky (1992) aplicaram 0; 100; 250 e $500 \mathrm{mg} \mathrm{planta}^{-1}$ de PBZ no substrato de cultivo de plântulas de laranjeira 'Valência' (Citrus sinensis (L.) Osbeck), com 90 dias de idade, e constataram que a concentração de $500 \mathrm{mg} \mathrm{planta}^{-1}$ reduziu a massa foliar específica em $18 \%$.

Neste experimento, as plantas tratadas com PBZ, na ausência de $\mathrm{GA}_{3}$, apresentaram folhas com a coloração verde mais intensa do que as controle ou tratadas com $\mathrm{GA}_{3}$, o que foi comprovado pelos resultados das unidades SPAD (Figura 3C).

Segundo Wood (1984), Sankhla et al. (1985) e Wang et al. (1986), na maioria dos casos, essa aparência está correlacionada ao aumento do teor de clorofila. Entretanto, há dúvidas se o aumento do teor de clorofila foi ocasionado pelo aumento da síntese de clorofila ou simplesmente por um "efeito concentrador" devido à reduzida expansão foliar (Davies \& Sankhla, 1987).

Os resultados encontrados neste experimento para unidades SPAD, nos tratamentos com PBZ, na ausência de GA, foram crescentes, apresentando valores médios que variaram de 53,537 unidades para o controle, a 62,379 , para a concentração de $225 \mathrm{mg} \mathrm{planta}^{-1}$, o que corresponde a um incremento na ordem de $16,52 \%$.

As concentrações crescentes de PBZ aumentaram linearmente as unidades SPAD, indicando haver relação entre PBZ e teor de clorofila nas folhas de limoeiro 'Volkameriano'. O $\mathrm{GA}_{3}$ neutralizou os efeitos do PBZ e promoveu maior crescimento das plantas, provocando "efeito diluidor" da clorofila e resultando em menores valores de leituras SPAD.

TABELA 1 - Resumo das análises de variância do comprimento de caule (CC), diâmetro de caule (DC), número de folhas (NF), comprimento de entrenó (CE), área foliar (AF), massa foliar específica (MFE) e unidades SPAD de plantas de limoeiro 'Volkameriano', avaliados aos 50 dias após o início dos tratamentos.

\begin{tabular}{ccccccccc}
\hline \multirow{2}{*}{$\begin{array}{l}\text { Fontes de } \\
\text { Variaçãa }\end{array}$} & \multirow{2}{*}{$\mathbf{G L}$} & $\mathbf{7}$ & $\mathbf{7}$ Quadrados Médios \\
\cline { 2 - 8 } & & $\mathbf{C C}$ & $\mathbf{D C}$ & $\mathbf{N F}$ & $\mathbf{C E}$ & $\mathbf{A F}$ & MFE & SPAD \\
\hline Bloco & 3 & 8,4446 & 0,0019 & 1,6245 & 0,0064 & 0,0003 & 597,3788 & 2,7460 \\
$\mathrm{PBZ}$ & 3 & $97,0240^{* *}$ & $0,0163^{* *}$ & $4,9545^{\mathrm{ns}}$ & $0,2878^{* *}$ & $6,5290^{* *}$ & $156,4934^{*}$ & $51,0460^{* *}$ \\
$\mathrm{AG}_{3}$ & 1 & $7338,6600^{* *}$ & $0,1099^{* *}$ & $219,4500^{* *}$ & $1,1223^{* *}$ & $10,7106^{* *}$ & $5288,9460^{* *}$ & $575,9600^{* *}$ \\
$\mathrm{PBZ} \mathrm{x} \mathrm{AG}_{3}$ & 3 & $378,8310^{* *}$ & $0,0082^{*}$ & $0,9845^{\text {ns }}$ & $0,1005^{* *}$ & $7,0575^{* *}$ & $316,3372^{* *}$ & $19,1559^{*}$ \\
Resíduo & 21 & 15,8230 & 0,0017 & 5,4731 & 0,0030 & 0,3537 & 50,9722 & 5,0338 \\
\hline $\mathrm{CV}(\%)$ & & 4,67 & 4,72 & 4,28 & 3,67 & 6,17 & 2,47 & 4,17 \\
\hline
\end{tabular}

** $F$, significativo a $1 \% ; * F$, significativo a $5 \%$; e ${ }^{\text {ns }} F$, não-significativo a $5 \%$. 


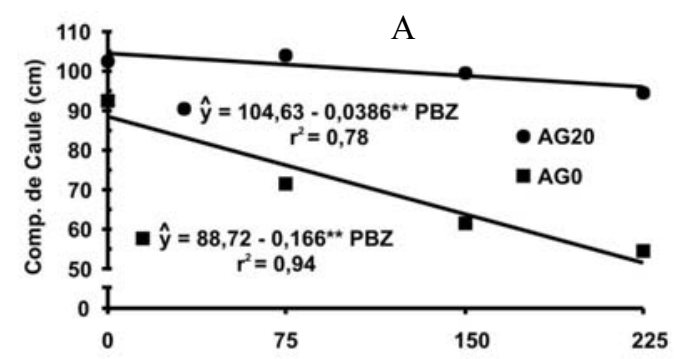

B



FIGURA 1 - Estimativa do comprimento (A) e diâmetro de caule (B) de plantas de limoeiro 'Volkameriano', em função das concentrações de $\mathrm{PBZ}$ na presença (AG20) ou ausência (AG0) de $\mathrm{GA}_{3}$, aos 50 dias após a aplicação dos produtos $\left(* \mathrm{e}^{* *}\right.$, significativos, a $5 \%$ e $1 \%$, pelo teste $t$, respectivamente).

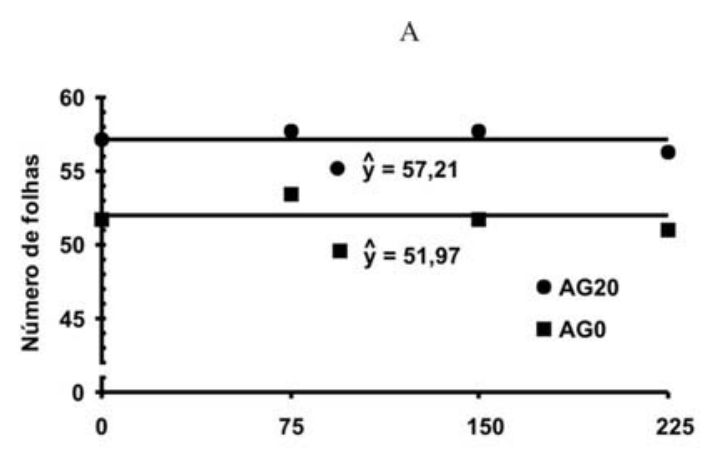

B

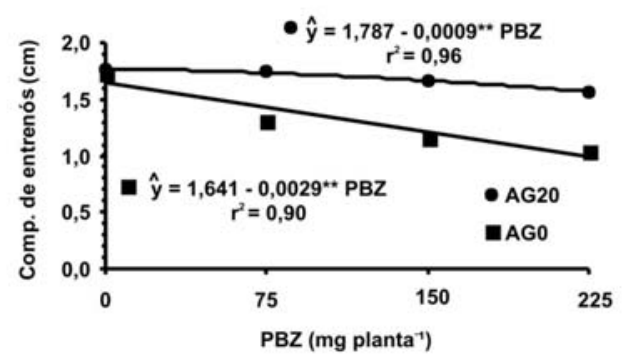

FIGURA 2 - Estimativa do número de folhas por planta (A) e comprimento de entrenós (B) de plantas de limoeiro 'Volkameriano', em função das concentrações de PBZ na presença (AG20) ou ausência (AG0) de $\mathrm{GA}_{3}$, aos 50 dias após a aplicação dos produtos $\left(* \mathrm{e}^{* *}\right.$, significativos, a $5 \%$ e $1 \%$, pelo teste $t$, respectivamente).

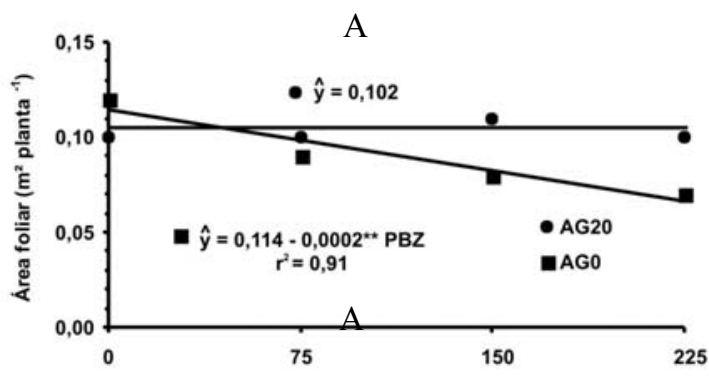

B
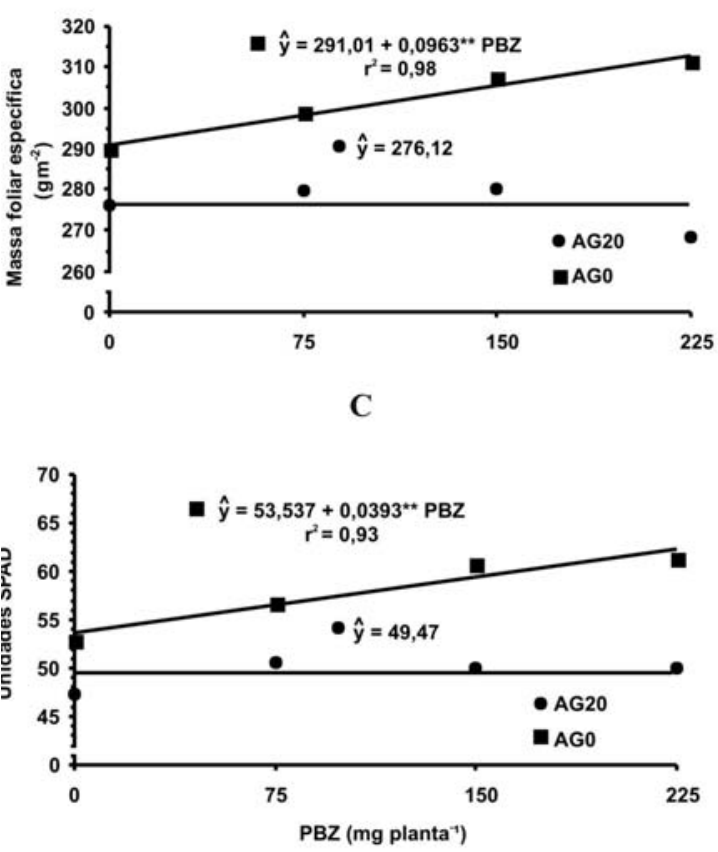

FIGURA 3 - Estimativa da área foliar (A), massa foliar específica (B) e unidades SPAD (C) de plantas de limoeiro 'Volkameriano', em função das concentrações de PBZ na presença (AG20) ou ausência (AG0) de $\mathrm{GA}_{3}$, aos 50 dias após a aplicação dos produtos $(* \mathrm{e} * *$, significativos, a $5 \%$ e $1 \%$, pelo teste $t$, respectivamente).

\section{CONCLUSÕES}

1-Concentrações crescentes do paclobutrazol (PBZ), na ausência de $\mathrm{GA}_{3}$, reduziram o desenvolvimento do caule, do limoeiro 'Volcameriano', manifestado por menores valores do comprimento e diâmetro do caule, e comprimento dos entrenós.

2-A aplicação do PBZ, na ausência de $\mathrm{GA}_{3}$, modificou algumas características de desenvolvimento das folhas do limoeiro 'Volkameriano', reduzindo a área foliar, aumentando a massa foliar específica e a intensidade da cor verde das folhas.

3-A aplicação de $\mathrm{GA}_{3}$ nas plantas, após a aplicação do PBZ, impediu que os efeitos do PBZ se manifestassem; portanto, o desenvolvimento vegetativo das plantas tratadas com ambos os fitorreguladores foi semelhante ao das plantas-testemunha.

4-A aplicação isolada de $\mathrm{GA}_{3}$, na dose de $20 \mathrm{mg}$ do 
ingrediente ativo $\mathrm{L}^{-1}$, aumentou o número de folhas do limoeiro Volkameriano em $10 \%$ e reduziu o comprimento do caule em $17,93 \%$.

\section{REFERÊNCIAS}

ARON, Y.; MONSELISE, S.P.; GOREN, R.; COSTO, J. Chemical control of vegetative growth in citrus trees by paclobutrazol. Hortscience, Alexandria, v.20, n.1, p.96-98, 1985.

BARRET, J.E.; BARTUSKA, C.A. PP333 effects on stem elongation dependent on site of application. HortScience, Alexandria, v.17, n.5, p.737-738, 1982.

BAUSHER, M.G.; YELENOSKY, G. Sensitivity of potted citrus plants to top sprays and soil applications of paclobutrazol. Hortscience, Alexandria, v.21, n.1, p.41-143, 1986.

BRAUN, J.W.; GARTH, J.K.L. Growth and fruiting of 'HeritGAe' primocane fruiting red raspberry in response to Paclobutrazol. HortScience, Alexandria, v.21, p.437-439, 1986.

DAVIES, T.D.; SANKHLA, N. Altered diurnal leaf movements in soybean seedlings treated with triazole growth regulators. Plant Cell Physiology, Kyoto, v.28, n.7, p.1345-1349, 1987.

DE JONG, T.M.; DOYLE, J.F. Leaf gas exchange and growth responses of mature 'Fantasia' nectarine trees to paclobutrazol. Journal of the American Society for Horticultural Science, Alexandria, v.109, p. 878-882, 1984.

DELGADO, R.; CASAMAYOR, R.; RODRIGUEZ, J.L.; CRUZ, P.; FAJARDO, R. Paclobutrazol effects on mandarin under tropical conditions. Acta Horticulturae, Wageningen, n. 179, 1986.

HADLOW, A.P.; ALLAN, P. Effect of paclobutrazol on vegetative growth in citrus nursery trees. South African Journal of Plant and Soil, Petroria, v.6 p.50-52, 1989.

JIAO, J.; TSUJITA, J.J.; MURR, D.P. Effects of paclobutrazol and A-Rest on growth, flowering, leaf carbohydrate, and leaf senescence in 'Nellie White' Easter lily (Lilium longiflorum Thunb.). Scientia Horticulturae, Amsterdan, v.30, n.1-2, p.135141,1986

LIDÓN, A.G.; BERNAL, I.M.; MARTÍNEZ, A.C.; FERNÁNDEZ, F.J.B.; CASTILLO, I.P. Influencia del Paclobutrazol em patrones de cítricos. Investigación Agraria. Producción y protección vegetales, Madrid, v.16, n.1, p.59-69, 2001.

MEHOUACHI, J.; TADEO, F.R.; ZARGAOZA, S.; PRIMOMILLO, E.; TALON, M. Effects of gibberellic acid and paclobutrazol on growth and carbohydrate accumulation in shoots and roots of citrus rootstock seedlings. Journal of Horticultural Science, Ashford, v.71, n.5, p. 747-754, 1996.
MONSELISE, S.P.; GOLDSCHMIDT, E.E. Alternative bearing in fruit trees. Horticultural Reviews, New York, v.4, p.128-173, 1982.

QUINLAN, J.D. New chemical approaches to control of fruit tree form and size. Acta Horticulturae, Wageningen, n.120, p.95-106, 1981.

RADEMACHER, W. Inhibitors of gibberellin biosynthesis: applications in GAriculture and horticulture. In: TAKAHASHI, N.; PHYNNEY, B.O.; MacMILLAN, J. (Ed.). Gibberellins. New York: Springer-Verlang, 1991.p.296-310.

SANKHLA, N.; DAVIS, T.D.; JOLLEY, V.D.; UPADHYAYA, A. Effect of Paclobutrazol on the development of iron chlorosis in soybeans. Journal of Plant Nutrition, New York, v.9, n.7, p.923934, 1986.

SANKHLA, N.; DAVIS, T.D.; UPADHYAYA, A.; SANKHLA, D.; WALSER, R.H.; SMITH, B.N. Growth and metabolism of soybean as affected by Paclobutrazol. Plant Cell Physiology, Kyoto, v.26, p.916-921, 1985.

SORGONÁ, A.; ABENAVOLI, M.R.; CACCO, R. A comparative study between two citrus rootstocks: effect of nitrate on the root morpho-topology and net nitrate uptake. Plant and Soil, Rockville, v. 270, p.257-267, 2005.

STEFFENS, G.L.; BYUN, J.K.; WANG, S.Y. Controlling plant growth via the gibberellin biosynthesis - I. Growth parameter alterations in apple seedlings. Physiologia Plantarum, Copenhagen, v.63, n.2, p.163-168, 1985.

SWIETLIK, D.; MILLER, S.S. The effect of Paclobutrazol on growth and response to water stress of apple seedlings. Journal of the American Society for Horticultural Science, Alexandria, v.108, n.6, p.1076-1080, 1983.

TUKEY, L. D. Vegetative control and fruiting on mature apple trees treated with PP333. Acta Horticulturae, Wageningen, n.137, p.103-109, 1983.

VU, J.C.V.; YELENOSKY, G. Growth and photosynthesis of sweet orange plants treated with Paclobutrazol. Journal of Plant Growth Regulation, New York, v.11, p.85-89, 1992.

WANG, S.Y.; SUN, T.; FAUST, M. Translocation of paclobutrazol, a gibberellin biosynthesis inhibitor, in apple seedlings. Plant Physiology, Rockville, v.82, n.1, p.11-14, 1986.

WILLIAMSON, J.G.; COSTON, D.C.; GRIMES, L.W. Growth response of peach roots and shoots to soil and foliar-applied paclobutrazol. HortScience, Alexandria, v.21, p.1001-1003, 1986. 